\title{
RILIEVO ED ANALISI URBANA: 25 ANNI DI COOPERAZIONE INTER-UNIVERSITARIA
}

Roberto Mingucci ${ }^{1}$

Questo breve contributo, introduttivo ad un volume interamente dedicato ad una speciale edizione della Summer School "Sviluppo urbano e cooperazione internazionale" (che con il decisivo sostegno del Dr Arturo Alberti, Presidente della Fondazione AVSI, da quattro anni realizziamo come iniziativa ufficiale dell'Ateneo di Bologna) non potrà non essere colmo di riferimenti personali, in particolare, di un'attività iniziata attorno ad un progetto della ONG AVSI (continuazione per molti versi di una tesi da me sviluppata per la laurea in Ingegneria edile presso la Facoltà di Bologna).

L'aspetto che tenterò di mettere qui in evidenza riguarda quindi non tanto il metodo di lavoro seguito nelle varie fasi realizzate in quest'attività venticinquennale, ma il carattere e l'importanza del coinvolgimento personale e dell'amicizia che, estendendosi nel tempo ad un ampio numero di persone, I'hanno, di fatto, resa possibile e produttiva.

\section{3 - nuove tecnologie ed avvio di una collaborazione}

L'approvazione di una innovativa legge municipale, la legge ProFavela, ${ }^{2}$ deliberata dal Consiglio municipale di Belo Horizonte dopo una lunga mobilitazione degli aderenti alla Pastorale di favela e alle associazioni sindacali degli abitanti, aveva fornito lo strumento per ipotizzare interventi di risanamento e conservazione delle aree di favela della città. Tali aree, in cui molte famiglie povere avevano investito lavoro e risparmi per realizzare un primo embrione di abitazione, che fino ad allora erano totalmente illegali e spesso rase al suolo da interventi pubblici, da quel momento diventavano zona SE4 del Piano Direttore e considerate come possibili aree di intervento per una riqualificazione urbana $e$, ancora più determinante, aree che poteva essere assegnate in proprietà a chi le aveva in uso come unica abitazione della propria famiglia.

Troppo lungo sarebbe tentare di ricostruire, anche sinteticamente, tutte le tappe che l'attuazione di tale legge ha comportato nel municipio di Belo Horizonte ${ }^{2}$ ma val la pena di ricordare che, in ragione della scelta effettuata dalle norme di attuazione della legge, di documentare con rilevi in scala 1:250 i piccoli lotti delle varie agglomerazioni allora censite, si intuì come assolutamente utile se non proprio indispensabile, l'utilizzo di stazioni topografiche totali che avrebbero consentito di sviluppare il lavoro di rilievo topografico in tempi e con costi più accettabili.

L'avvio di un programma quadro che aveva già portato a Belo Horizonte due Cooperanti della ONG $\mathrm{AVSI}^{4}$ consentì di ampliare le
1. Ingegnere (Unibo), Professore Ordinario di Disegno dell'Università di Bologna.

2. La legge $N^{\circ} 3532$ del 6 Gennaio 1983 che di fatto ha avviato il grande dibattito del recupero delle aree marginali e l'affermazione del principio da sempre sostenuto dai favelados del Brasile " La favela non è un problema ma l'inizio di una soluzione"
3. E' sperabile che lo possano fare ricercatori e laureandi della Facoltà di Architettura della PUC, che in qualche modo ha radici ed origini concretamente collegate a quell'esperienza.

4. Si trattava dei coniugi Anna e Livio Michelini che fondamentale importanza ebbero poi nel prosieguo dei progetti AVSI prima a BH - Minas Gerais e poi a Salvador - Bahia

Cadernos de Arquitetura e Urbanismo, v.16, n.18+19, 2009 
attività previste in quel programma e di avviare la collaborazione formale fra PUC e Università di Bologna. ${ }^{5}$

I rapporti tra il Dipartimento di Architettura e Pianificazione Territoriale dell'Università di Bologna e la Pontificia Università Cattolica di Minas Gerais (Belo Horizonte - Brasile) risalgono quindi al 1983, anno del primo viaggio effettuato a Belo Horizonte da me e dal Dr. Arturo Alberti, quando I'incontro con il Prof João Francisco De Abreu, allora pro-rettore per le relazioni internazionali, segnò non solo l'avvio delle attività di cooperazione ma l'inizio di un'amicizia così cordiale e gratuita, che avrebbe nel tempo contagiato molti nostri colleghi e studenti di Corsi di specializzazione post-laurea, organizzati in collaborazione fra le nostre due istituzioni, oggi professionisti e docenti sia alla PUC sia in altre università di $\mathrm{BH}$ e del Brasile. ${ }^{6}$

\section{Corsi post-laurea, e la collaborazione nei master e nei dottorati}

La tematica del recupero delle aree di favela non incontrava negli atenei brasiliani un naturale interesse e solo in ragione di un dialogo iniziato fra le persone coinvolte coi progetti AVSI di fatto riuscì a porre anche all'attenzione del mondo accademico questa particolare area di ricerca, sia nei suoi contenuti tecnici sia nel suo più profondo significato politico-culturale. ${ }^{7}$

Dopo questa esperienza di formazione e ricerca, sviluppata fra il 1988 ed il 1995, dal 1996 la PUC ha dato avvio ad uno specifico programma post-laurea, nell'ambito della facoltà di Geografia, attraverso l'istituzione di un master e successivamente di un dottorato, con particolare riferimento alle linee di ricerca sui temi delle scienze regionali e del supporto delle tecnologie dell'informazione geografica alla costruzione del quadro conoscitivo in ambito urbano e territoriale. Sono cominciati quindi, tra il DAPT ed il Programma Post-Laurea della PUC in "Tratamento da Informação Espacial", ulteriori scambi di docenti, neo-laureati e dottorandi in attività seminariali, didattiche e di ricerca in tali settori disciplinari.

In particolare tali attività sono consistite nella partecipazione di Docenti, ricercatori e dottorandi in convegni organizzati dalla PUC e dall'Università di Bologna ${ }^{8}$ nell'attività didattica nella disciplina dei sistemi informativi geografici, nell'ambito del master e dottorato in "Tratamento da Informação Espacial" della PUC ed in scambi di neo-laureati e ricercatori italiani e brasiliani per brevi periodi di ricerca sui temi citati in precedenza.

In quest'ultimo periodo la collaborazione tra le Università di Bologna e la PUC si è ulteriormente ampliata, arrivando a formalizzare una convenzione fra i due atenei (sottoscritta dai rispettivi Rettori) che ha potenzialmente aperto la strada anche a collaborazioni didattiche e di ricerca in nuovi settori, e si è di fatto riattivata sia su nuovi progetti di ricerca (che riprendono i temi della legalizzazione e del recupero delle aree urbane informali) sia sul piano della formazione attraverso alcune iniziative collegate (limitate al momento a particolari tematiche) per il perfezionamento di dottorandi e di stage di post-dottorato. ${ }^{9}$
5. Fu questa l'occasione infatti di un primo programma di cooperazione per la formazione di un topografo, già formato presso la PUC, che venne il Italia per uno stage presso la nostra Facoltà di Ingegneria e presso uno studio professionale di Rovereto che già utilizzava la tecnologia prescelta. L'ing. Nonato Saraiva coinvolto in questa prima fase, a Bologna fu accolto dall'allora Istituto di Architettura ed Urbanistica e da quello di Topografia, lavorando, oltre che con me, con i Proff. Marco Ungendoli e Giuseppe Lombardini, che si erano resi disponibili a collaborare al progetto. L'ing. Nonato si trasferì poi a Rovereto, per una esperienza pratica sul Campo organizzata da Sergio Faccioli e dai colleghi dello studio S126.

6. A tal proposito si possono ricordare i corsi svolti negli anni 1989 (Programmi di intervento in aree marginali urbane) e 1994 (Corso di Specializzazione Post Laurea in Ecologia Urbana) tenuti a Belo Horizonte presso la stessa PUC da docenti delle due università, a gruppi selezionati di circa 20 allievi per ogni corso, seguiti da stage in Italia svolti in specifici ambiti disciplinari e tecnologici quali il rilievo topografico automatizzato di aree urbane ed i sistemi informativi geografici (GIS) per progetti di recupero di aree urbane informali in Brasile.

7. A tal proposito si possono ricordare i corsi svolti negli anni 1989 (Programmi di intervento in aree marginali urbane) e 1994 (Corso di Specializzazione Post Laurea in Ecologia Urbana) tenuti a Belo Horizonte presso la stessa PUC da docenti delle due università, a gruppi selezionati di circa 20 allievi per ogni corso, seguiti da stage in Italia svolti in specifici ambiti disciplinari e tecnologici quali il rilievo topografico automatizzato di aree urbane ed i sistemi informativi geografici (GIS) per progetti di recupero di aree urbane informali in Brasile.

8. Ad esempio il "III Encontro da Associação Brasileira de Estudos Regionais (ABER) - Pobreza, Desenvolvimento Humano e Desigualdades Regionais", Belo Horizonte - MG - Brasil 20 - 22 Giugno 2004 ed il Seminario internazionale "La qualità dello spazio: dal piano al progetto", Cesena 18-19 Dicembre 2003.

9. In particolare tali attività sono state sviluppate sui temi della pianificazione dei bacini idrografici con particolare riferimento al supporto delle tecnologie dell'informazione geografica (dott. Aurelio Muzzarelli, Prof. Joao Francisco de Abreu, Prof. Piero Secondini) e di post-dottori di ricerca (Prof. Arch. Altino Barbosa Caldeira, Prof. Roberto Mingucci, Prof. Fabrizio I. Appollonio, Ing. Giuseppe Amoruso) sui temi del rilievo e della catalogazione dei beni architettonici nel contesto urbanistico e territoriale. 


\section{Il futuro di questa collaborazione}

La consistenza, durata e qualità del rapporto tra il DAPT dell'Università di Bologna e la PUC (in particolare i Dipartimenti di Geografia ed Architettura) hanno garantito la possibilità di avviare un rapporto stabile tra i due Atenei, oggi infatti regolato da una specifica convenzione.

In modo analogo a quanto già avviene con altri Atenei brasiliani (ad esempio la stessa UFMG - Università Federale del Minas Gerais) si vuol verificare la possibilità di istituire un inter-dottorato (tra il DAPT che sviluppa un dottorato in "Ingegneria Edilizia eTerritoriale" e la Scuola di dottorato in "Tratamento da Informação Espacial" della PUC) che consenta ai giovani coinvolti di realizzare una formazione accademica riconosciuta da entrambi gli stati. Non solo, infatti, appare evidente il grande interesse su temi di ricerca che potrebbero essere sviluppati (dal recupero architettonico ed urbanistico, alla pianificazione urbanistica e territoriale, al supporto delle tecnologie del rilievo alla scala architettonica ed urbana, alla ricerca sulle tecnologie dell'informazione geografica distribuite in rete) ma soprattutto risulta confermato dall'esperienza trascorsa che queste modalità di collaborazione internazionale costituiscono occasioni irripetibili ed efficaci per l'avvio e la costruzione di iniziative di reale cooperazione culturale che sono alla base sia della crescita delle persone che le realizzano sia dello sviluppo sociale delle istituzioni coinvolte. 